Postprint manuscript for self-archiving purposes.

PHYSICAL REVIEW B 93, 235307 (2016)

DOI: http://dx.doi.org/10.1103/PhysRevB.93.235307

\title{
Spherical silicon photonic microcavities: From amorphous to poly-crystalline
}

\author{
R. Fenollosa ${ }^{1, *}$, M. Garín ${ }^{2}$, F. Meseguer ${ }^{1}$ \\ ${ }^{1}$ Instituto de Tecnología Química (CSIC - UPV), Universitat Politècnica de Valencia, \\ Av. Tarongers $s / n$ 46022, Valencia, Spain \\ ${ }^{2}$ Grup de recerca en Micro i Nanotecnologies, Departament d'Enginyeria Electrònica, \\ Universitat Politècnica de Catalunya, c/ Jordi Girona 1-3, Barcelona 08034, Spain \\ *rfenollo@ter.upv.es
}

Shaping silicon as a spherical object is not an obvious task, specially when the object size is in the micrometer range. This has the important consequence of transforming bare silicon material in a microcavity, able to confine light efficiently. Here, we have explored the inside volume of such microcavities, both in their amorphous and in their poly-crystalline versions. The synthesis method, which is based on chemical vapour deposition, causes amorphous microspheres to have a high content of hydrogen that produces an onion-like distributed porous core when the microspheres are crystallized by a fast annealing regime. This influences substantially the resonant modes. However a slow crystallization regime does not yield pores, and produces higher quality factor resonances that could be fitted to Mie theory. This allows establishing a procedure for obtaining size calibration standards with relative errors of the order of $0.1 \%$.

PACS number(s): 78.20.Bh, 78.20.Ci, 78.67.-n 


\section{INTRODUCTION}

Silicon microspheres constitute a promising platform for developing applications in many technological disciplines [1-5]. Their main strength stems from their capability to sustain optical resonances, i.e. of being photonic microcavities, and the fact that they are made of silicon, which is, among other things, the material basis of the current microelectronics industry. Silicon micro and nanoparticles have been traditionally considered as a by-product in the context of thin film synthesis by Chemical Vapour Deposition (CVD) [6,7]. For instance, one can read in silicon technology books [8] about the appearance of a sort of unwanted "silicon soot" during the synthesis of silicon by the Siemens method. However, in the last years silicon microspheres have received a growing attention from researchers working in photonics and metamaterials [1-5,9-14]. At the same time, different fabrication methods have arisen [3,9,15-18].

The synthesis route based on CVD, using di-silane $\left(\mathrm{Si}_{2} \mathrm{H}_{6}\right)$ as precursor gas [9], is very convenient because it allows the fabrication of both phases, amorphous and poly-crystalline, due to the relatively low decomposition temperature (around 400 ${ }^{\circ} \mathrm{C}$ ) of the precursor. In other words, amorphous microspheres can be synthesized at low decomposition temperatures (375-600 ${ }^{\circ} \mathrm{C}$ ), while poly-crystalline spheres can be produced by using higher process temperatures. Poly-crystalline microspheres can be also obtained from their amorphous counterparts by a subsequent annealing process. However, the high hydrogen content of the precursor translates into the chemical composition of the microspheres, what may produce strong disturbances during the crystallization process in the form of voids, thus strongly influencing their optical and electrical properties. So far, the optical properties of such microspheres have been usually characterized by optical transmittance spectroscopy. This requires transferring them to a low refractive index substrate, like glass. In this way, the optical resonance phenomenon was verified and the experimental peaks could be associated to Mie resonances $[9,19]$. This characterization has been accomplished for low size parameters (defined as $\pi d / \lambda$, where $d$ is the sphere diameter and $\lambda$ is the wavelength of light), where the resonant peaks are relatively wide and weakly influenced by any defects in the particles. However, the most recent applications based on this material [14], as well as any future development, require an in depth structural and optical characterization. This is very important if we want to push silicon microspheres to a more prominent technological position, e.g. being integrated in photonic/optoelectronic chips, or in other fields such as medicine, where silicon microspheres have already been shown to be biocompatible [20] and potential active agents for cancer therapy [21].

Here we report a substantial step forward in characterizing and understanding the relation between structural and optical properties of such silicon microspheres. In particular we have studied the influence of the crystallization process on their internal structure and on their optical properties. This has required the development of techniques for measuring the optical resonances at higher size 
parameters, avoiding the influence of the substrate. Such resonances are more pronounced, i.e. with a higher quality factor, than those occurring at low size parameters. They are, therefore, much more sensitive to the defects in the material. The obtained results have revealed interesting opportunities for the development of new applications. For instance, we have realized that a fast crystallization regime produces particles with a richly structured porous core, whereas a low crystallization regime does not. Such porous core is not necessarily a drawback because, although it kills some resonances and might lead to a poor electrical quality, it can be utilized for future developments requiring the introduction of other substances into the pores. Also, it can be used to control (filter) the resonances present in the particle. Finally, the very small errors for the sphere diameter values obtained through fitting of the scattering spectra to Mie theory, suggest proposing silicon microspheres as size calibration standards for microscopy.

\section{EXPERIMENTAL}

\section{A. Synthesis and crystallization processes of silicon microspheres}

Silicon microspheres were synthesized by decomposing di-silane gas $\left(\mathrm{Si}_{2} \mathrm{H}_{6}\right)$ in a closed reactor under controlled conditions of pressure and temperature [9]. The process parameters were optimized for producing a thin layer of amorphous microspheres, with size in the range 1-4 $\mu \mathrm{m}$, which are separated from one another[14]. This is very convenient because it allows us to identify them before and after any processing step, and optical measurements can be performed onto single particles without manipulating them, as it will be shown below. In all the cases, polished silicon slabs were used as carrier substrates onto which microspheres were directly synthesized and measured.

Amorphous silicon microspheres underwent different crystallization treatments at $800^{\circ} \mathrm{C}$ using a home-made horizontal quartz tube furnace. First of all we performed a short dip in HF solution (1\%) to the substrates containing the microspheres in order to remove the native oxide layer. Right after, the samples were introduced in the quartz tube, which was placed inside the tubular oven and the ambient was pumped out. Before the crystallization process, the samples were heated in vacuum at $200{ }^{\circ} \mathrm{C}$ during 1 hour in order to remove any traces of water. Then, the quartz tube was filled with nitrogen, without breaking the vacuum, at a pressure of about 2 atm and the crystallization annealing was carried out. Two different crystallization regimes were studied: a fast one and a slow one, whose temperature profiles are detailed in Fig. 1. 

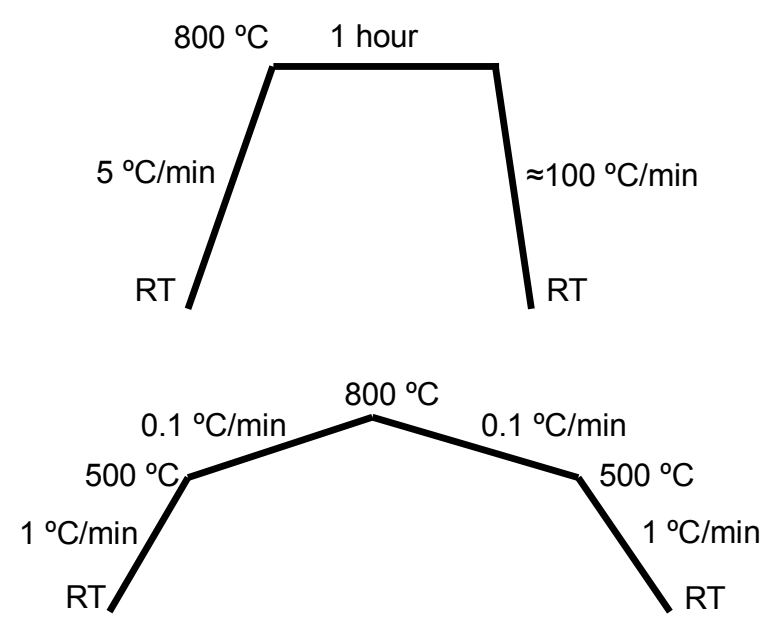

FIG. 1. Schematic of the two temperature profiles, fast (above) and slow (below), used for crystallizing amorphous silicon microspheres.

In the fast regime, the samples were heated at a rate of $5{ }^{\circ} \mathrm{C}$ per minute up to $800{ }^{\circ} \mathrm{C}$ and they were kept at this temperature during 1 hour. Then, the process was abruptly stopped by removing the quartz tube from the tubular oven directly into the room temperature environment. This is the usual crystallization regime we had been using so far. In the slow regime, the samples were heated at a speed of 1 ${ }^{\circ} \mathrm{C}$ per minute up to $500^{\circ} \mathrm{C}$, and at $0.1^{\circ} \mathrm{C}$ from this temperature up to $800^{\circ} \mathrm{C}$. Then the temperature decreased again to $500{ }^{\circ} \mathrm{C}$ at $0.1{ }^{\circ} \mathrm{C}$ per minute, and to RT from here at $1^{\circ} \mathrm{C}$ per minute. These characteristics were chosen for allowing hydrogen degassing at the crystallization point, which we assumed to occur at a temperature around $600{ }^{\circ} \mathrm{C}[22,23]$. In case of technological implementation of this method, more research could be focused in determining accurately the transition point and shorten the whole process.

\section{B. Optical resonances characterization}

Silicon microspheres yield optical resonances in the visible and near infrared ranges, which have been measured so far by optical transmittance spectroscopy $[9,19,24]$. Here we report their detection by optical scattering at $90^{\circ}$. This technique has been used for measuring resonances in spherical cavities which are several tens of micrometers in size, for example liquid droplets [25] and glass microspheres [26]. In our case, this approach is very advantageous because only those modes whose resonant plane occurs in the plane parallel to the substrate arrive to the detector. Here, this is of particular importance because the substrate is made of silicon and it is expected to kill the modes resonating in a plane crossing the substrate due to its high refractive index.

Figure 2 shows a scheme with the main elements of our measuring setup and, on the left side, a SEM image of a silicon microsphere placed on a substrate. The setup consists of a home-made confocal system where white light, indicated by the (red) grey areas, is focused onto a microsphere by a 20x objective lens with a NA of 
0.4. The scattered light at $90^{\circ}$ is collected by a similar objective and is focused on an iris that allows passing only the light that exits the sphere tangentially within a limited area, which has been indicated in the SEM image by a black line circle. This is an essential element for avoiding spurious light and improving the sensitivity of the system. However, it limits the detection to light coming from a particular area and direction, and some elements of the scattered light may be lost. This will be further commented later on in the framework of the obtained results. After having crossed the iris the light is divided by a beam splitter, where $10 \%$ of it goes to a camera for visualizing the position of the sphere and the scattered light. Such scattering effect, recorded by the camera, has been superimposed to the SEM image in the figure, corresponding to the light regions. The other $90 \%$ of light transmitted through the beam splitter enters into a spectrometer (Horiba iHR320), with the help of a beam expander, where it is measured by means of both a liquid nitrogen refrigerated CCD 2D array and a InGaAs 1D array detectors, for visible and near infrared light respectively.
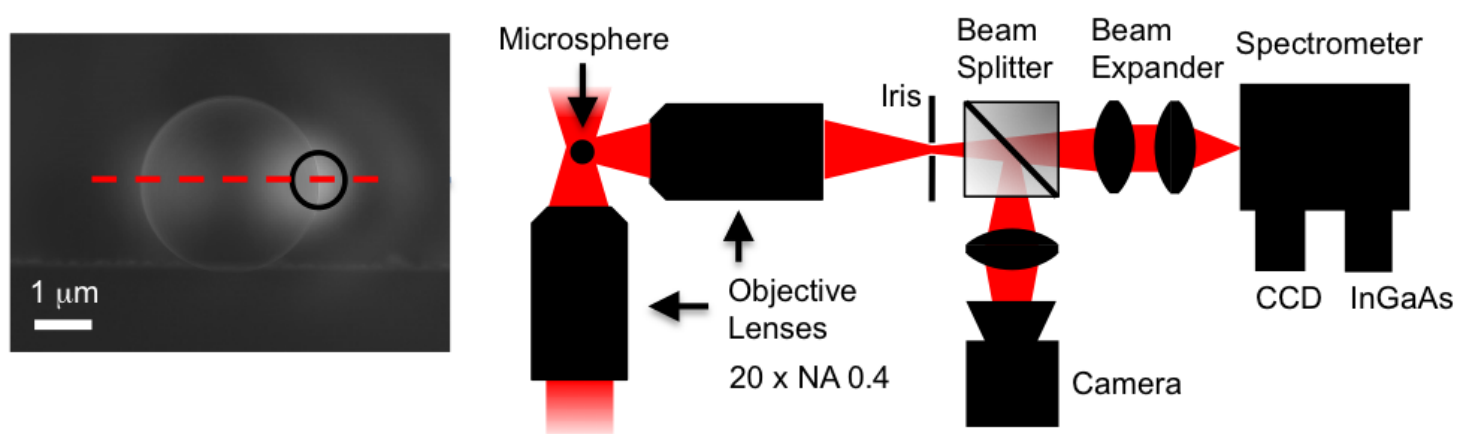

FIG. 2. (Color online) Scheme of the setup used for measuring the light scattering at $90^{\circ}$ of single silicon microspheres. The SEM image on the left side corresponds to a silicon microsphere lying on a substrate. The scattered light, as recorded by the camera, has been superimposed (light regions). The black line circle indicates the area of the Iris, projected on the microsphere, and the (red) dashed line indicates the position of the resonant plane for all the measured resonances.

All the measured spectra were corrected by the sensitivity of the system. For this purpose, the scattering produced by an unpolished silicon slab was used as a base curve. However, because the spectrometer sensitivity is highly dependent on the light polarization due to the diffraction gratings, and the particular polarization components of the scattered light are unpredictable, the corrected curves for the scattering spectra do not perfectly coincide with performed simulations as long as sensitivity is concerned, as it will be shown below. On the other hand, these discrepancies arise mostly at the longest wavelengths region of the spectra, where the sensitivity of the InGaAs detector decreases substantially. We are currently studying possible solutions to these problems. They include for instance the usage of light diffusers after the beam expander. 


\section{Structural characterization}

Structural properties of silicon microspheres were investigated by several electronic microscopy techniques. They include Field Emission Scanning Electron Microscopy (FESEM) for external aspects such as sphericity and surface smoothness, and High Resolution Transmission Electron Microscopy (HRTEM) for the internal structure. In the last case, microspheres were previously carved in planes perpendicular to the substrate, using a Focused Ion Beam (FIB), until obtaining several tens of nanometers thick micelles of the central part of the microspheres.

\section{RESULTS AND DISCUSSION}

\section{A. The influence of the crystallization regimes on the structural and optical properties of silicon microspheres: general observations.}

Amorphous silicon microspheres have very good conditions in terms of sphericity and surface smoothness, see for example Fig. 3 (a). These features, together with the high refractive index of silicon, are very promising for sustaining high quality factor, $Q$, optical resonances, where $Q$ can be calculated as the ratio between the resonant wavelength, $\lambda$, and the peak's full-width at half maximum, $\delta \lambda, Q=\lambda / \delta \lambda$. Because of these reasons, optical scattering spectra of amorphous silicon microspheres include many pronounced peaks whose origin is the optical resonance phenomena, as shown by the bottom curves of Fig.4 (a) and (b). They correspond to $Q$ values of the order of 1000. This value, however, is limited by the resolution and the sensitivity of the detection system and there may exist unmeasured higher $Q$ resonances beyond the resolution of the spectrometer. It will be commented more in detail in the next section. In any case, as expected the high $Q$ resonances disappear for wavelengths approaching the visible range because of the absorption of the material.
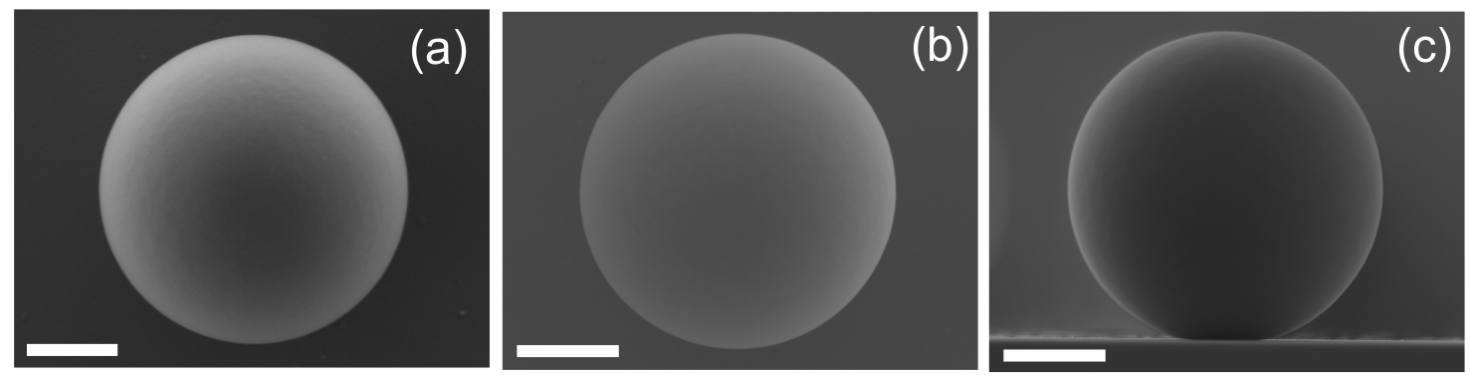

FIG. 3. FESEM images of a silicon microsphere (a) before, i.e. in amorphous state (top view), and (b), (c) after having been crystallized by using the fast regime (top and side view respectively). The scale bars correspond to $1 \mu \mathrm{m}$. 
The crystallization processes, under the conditions specified in section II.A, preserved the surface smoothness and the spherical shape of the particles for both regimes of crystallization. Figure 3 shows FESEM images of the same microsphere before, (a) (top view), and after having been crystallized by the fast regime, (b) (top view) and (c) (side view). We did not find any conclusive difference before and after the crystallization processes for these features within the limitation of the microscope resolution. However, in general it was observed a decrease of the sphere diameter, about $5 \%$ of the initial value, in the case of microspheres treated by using the slow crystallization regime. Table I shows the diameter of several microspheres before, and after crystallization by the slow regime. The values shown in the second and third columns correspond to diameters obtained from FESEM images. The last column shows the diameters of the same crystallized microspheres obtained by fitting their scattering spectra to Mie theory. This will be further discussed in the next section.

TABLE I. Diameter values in $\mathrm{nm}$ of several silicon microspheres before (amorphous) and after (crystallized) annealing by the slow regime. The second and third columns represent the values as measured from their FESEM images. The last column shows the diameters of the crystallized microspheres as obtained from the fit of their optical scattering spectra to Mie theory.

\begin{tabular}{cccc}
\hline \hline sphere \# & $\begin{array}{c}\text { amorphous } \\
(\text { FESEM) }\end{array}$ & $\begin{array}{c}\text { crystallized } \\
(\text { FESEM) }\end{array}$ & crystallized (fitting) \\
\hline 1 & $2810 \pm 20$ & $2670 \pm 20$ & $2681 \pm 5$ \\
2 & $2940 \pm 20$ & $2820 \pm 20$ & $2801 \pm 6$ \\
3 & $2520 \pm 20$ & $2410 \pm 20$ & $2406 \pm 6$ \\
4 & $2690 \pm 20$ & $2550 \pm 20$ & $2555 \pm 7$ \\
5 & $2930 \pm 20$ & $2780 \pm 20$ & $2786 \pm 6$ \\
6 & $2970 \pm 20$ & $2810 \pm 20$ & $2826 \pm 6$ \\
7 & $2800 \pm 20$ & $2670 \pm 20$ & $2676 \pm 6$ \\
8 & $3610 \pm 20$ & $3410 \pm 20$ & $3430 \pm 5$ \\
\hline \hline
\end{tabular}

Contrary to the structural properties of the surface, which remained unchanged, the optical scattering spectra of amorphous silicon microspheres were substantially modified after the crystallization processes. Figure 4 (a) shows two spectra of a microsphere, of about $3300 \mathrm{~nm}$ in diameter, before (bottom curve) and after (top curve) having been crystallized following the fast regime. This crystallization regime produces the disappearance of the sharp resonances. Only a coarse ripple structure and some relatively low $Q$-value (say about 300 ) resonant modes still remain in the scattering spectrum. Some differences between these curves could be expected in relation to the refractive index difference between amorphous and crystalline silicon, but not the disappearance of the sharp peaks. On the other hand, the slow crystallization regime was found to preserve higher $Q$ modes, as it is shown by Fig. 4 (b) for sphere \#6 in Table I. The difference between these two crystallization regimes must be, therefore, related to internal structure differences between the particles, as we will prove later through TEM imaging. 

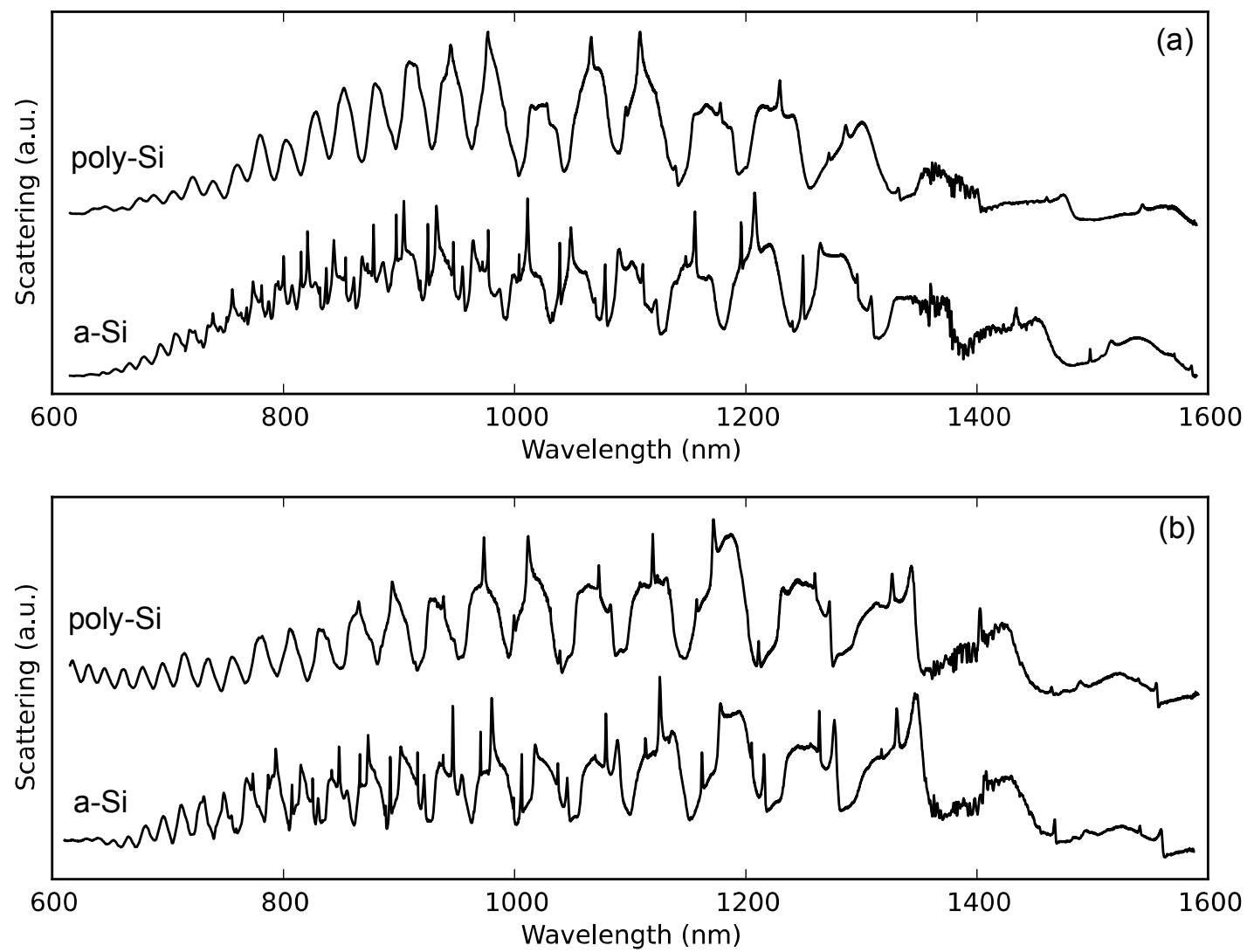

FIG. 4. (a) Scattering spectra of a silicon microsphere in amorphous state (bottom curve) and after having been treated by the fast crystallization regime (top curve). (b) Same as (a), but for the slow crystallization regime.

\section{B. Mie theory fit of spectra}

\section{Amorphous silicon microspheres}

Optical scattering spectra of amorphous silicon microspheres were fitted to theoretical spectra, calculated by Mie theory [27]. For this purpose we used the subroutines by Barber [28], for calculating the scattering of light by a spherical particle at $90^{\circ}$, and corrected the spectra to account for the NA of the objective lenses. The sphere diameter values measured by FESEM (Table I) were considered as starting parameters. Because of the uncertainty on the exact dispersion curve for amorphous silicon, which depends on the fabrication conditions, the refractive index was also adjusted along the fitting process at several points in intervals of $100 \mathrm{~nm}$, assuming linearly interpolated values between them. 
Figure 5 shows the good agreement obtained between the experimental (black curve) and the simulated ((red) grey curve) scattering spectrum for sphere \#6 of Table I, considering arbitrary values of offset and contrast so that the two curves overlap. Figure 6 corresponds to a zoomed spectral region of Fig. 5 showing in more detail few typical resonances. They have been labelled using the common notation [29] by letters ' $b$ ' and 'a' for transversal electric and transversal magnetic modes, respectively, followed by two indexes: the mode number, which indicates the number of electric field intensity maximums in the half sphere perimeter, and the order of the resonance, which accounts for the number of maximums in the radial direction respectively. The discrepancies in intensity and contrast between the simulated and the experimental spectra may have different causes, such as the uncertainty in the sensitivity correction (see section II.B) and the fact that the scattered light is measured in a reduced area at the microsphere's edge (see the black line circle at the SEM image of Fig. 2), and some components may be poorly detected, thus highlighting others. This could be the case, for instance, of resonance $\mathrm{a}_{12,5}$.

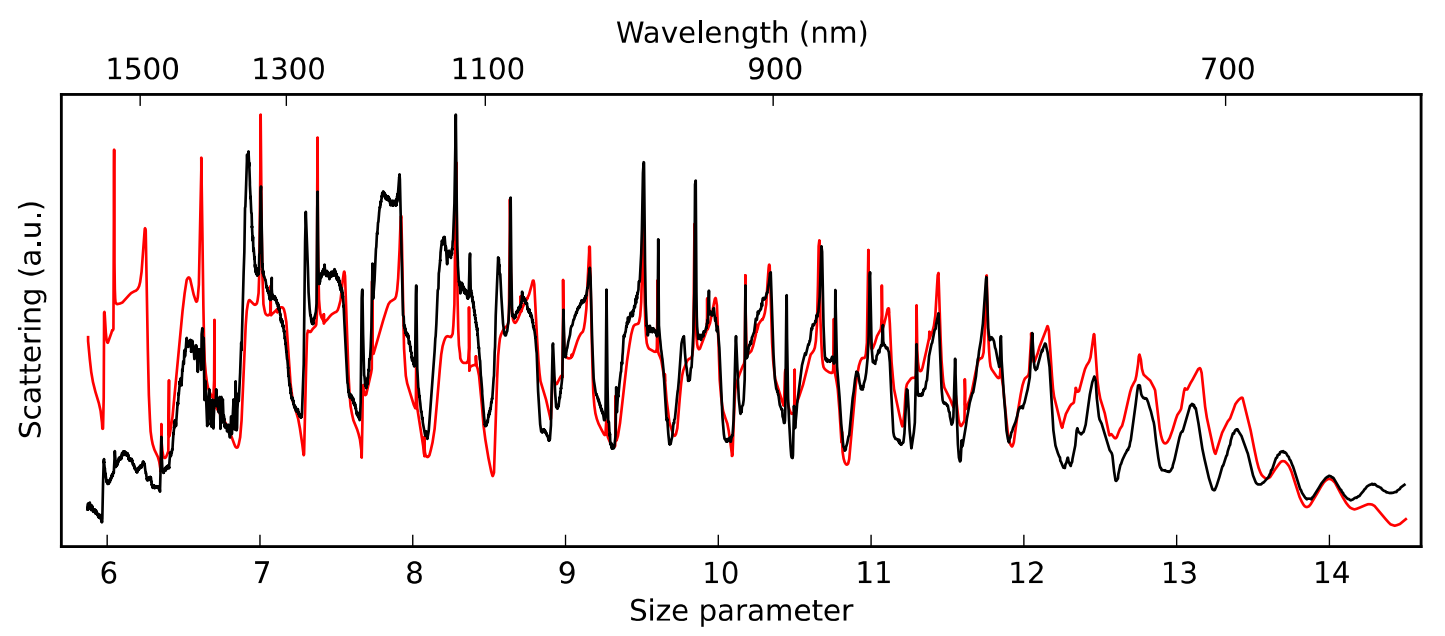

FIG. 5. (Color online) Experimental (black curve), and simulated ((red) grey curve) spectra for microsphere $\# 6$ of Table I in the amorphous state. The spectra are plotted against the wavelength of light, $\lambda$ (top axis) and the size parameter (bottom axis), defined as $\pi \mathrm{d} / \lambda$, where $\mathrm{d}$ is the sphere diameter. 


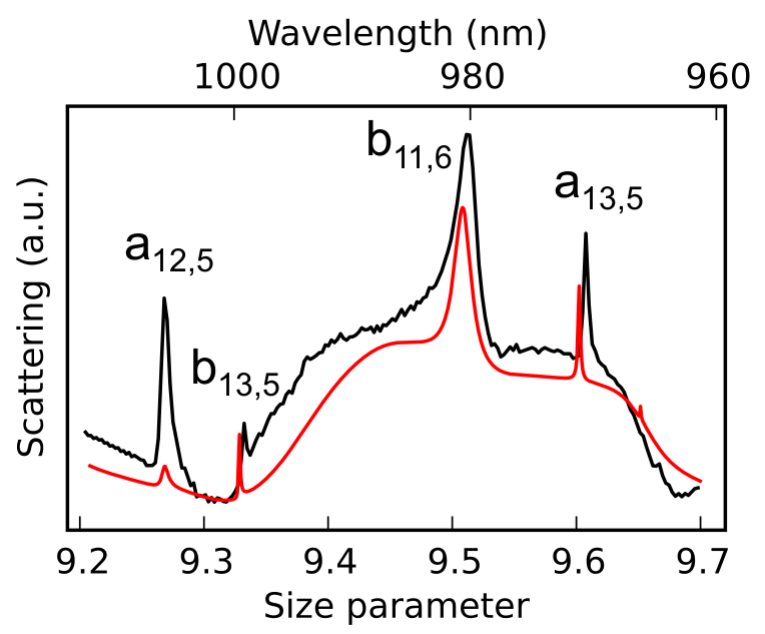

FIG. 6. (Color online) Reduced range of Fig. 5, where some typical resonances are indicated.

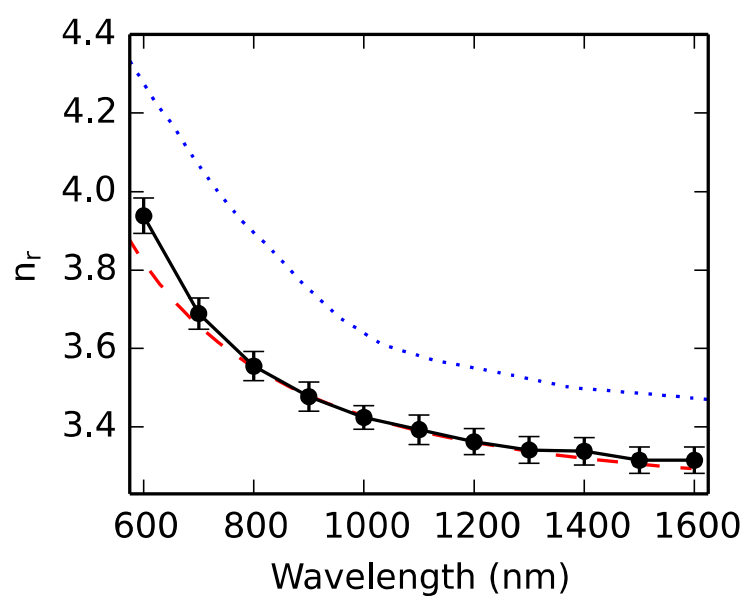

FIG. 7. (Color online) Real part of the refractive index dispersion utilized for fitting the scattering spectra of Fig. 5 (black curve and points). Other refractive index values corresponding to amorphous silicon reported in refs. 30 and 32, have been plotted for comparison as (red) dashed and (blue) dotted curves respectively.

The resulting fitted real part of the refractive index, $\mathrm{n}_{\mathrm{r}}$, is shown in Fig. 7 (black curve and points). Here, the error bars were calculated by fitting the scattering spectrum for the minimum and the maximum diameter values given by the error of the diameter (see second column in Table I). Furthermore, all the microspheres of Table I yielded refractive index values within these error bars. It is interesting to compare these values with others reported in the literature. We found that the refractive index dispersion of our amorphous silicon microspheres is very similar to that of hydrogenated microcrystalline silicon thin films reported by R.I. Brandan et al. [30], obtained by plasma enhanced chemical vapour deposition. The agreement is best for those samples with low crystalline volume fraction. The (red) dashed curve corresponds to their sample 5, which had a crystalline volume 
fraction of $68 \%$. These values are, however, much lower than other reference values of the refractive index collected by Palik [31], such as those of Pierce et al. [32] ((blue) dotted curve). This is not contradictory because there should be a substantial difference in the hydrogen content, which strongly depends on the synthesis method used.

The imaginary part of the refractive index, $n_{\mathrm{i}}$, originates from light absorption losses in the material but it can also include other spurious scattering effects such as surface roughness and defects. Therefore, $n_{\mathrm{i}}$ strongly influences both the height and width of the resonant peaks. In general, the higher the $n_{\mathrm{i}}$ the lower the scattering height, since light gets resonantly absorbed rather scattered, and the wider the resonance, due to the reduced $Q$. Eventually, for very high $n_{\mathrm{i}}$ values the resonances get killed [33]. The fit of Fig. 5 was achieved by considering $n_{\mathrm{i}}$ values of $0.06,0.004$ and 0.0001 for wavelengths of 600,700 and $800 \mathrm{~nm}$ respectively, with linear interpolation between these points, and an arbitrary constant value of 0.0001 for wavelengths higher than $800 \mathrm{~nm}$. These values, however, should be considered as a qualitative approach because of the limited resolution, around 1 $\mathrm{nm}$, of the measurement, what imposes a limit to the highest measurable $Q$ of about 1000 .

\section{Crystallized silicon microspheres}

Figure 4 (a) showed how most of the pronounced peaks disappeared from the scattering spectrum after the fast crystallization process. Such disappearing peaks correspond to Mie resonances with high order numbers, i.e. modes with several electric field intensity maximums in the radial direction, as it has been shown in Fig. 6. They are well distributed inside the microspheres; therefore, in the absence of surface defects, structural defects localized inside them are expected to strongly influence such resonances, effectively killing them. This hypothesis was confirmed by the structural measurements of the inner part of the microspheres. Figure 8 (a) shows a HRTEM image of the internal structure of a fast crystallized sphere of about 3.5 micrometers in diameter superimposed, as an example, by the electric field intensity distribution of mode $b_{13,5}$. As it can be observed in the image, the fast crystallization process produces an inner porous structure surrounded by a nonporous shell (NPS). The existence of pores inside crystallized silicon microspheres had already been reported [14], but it had not been studied in as much detail as we do here. The pore size distribution is highly dependent on the radial direction and can be divided into three parts, indicated in the figure as P1, P2 and P3. Figure 8 (b) corresponds to a zoomed area of Fig. 8 (a), and Fig. 8 (c) and (d) are higher magnification images that allow a better visualization of such porous regions, as well as the transitions between them. The innermost part, contained within a radius of 1 micrometer ( $\mathrm{P} 1$ region), has pores with undefined shape, and size in the range of $100 \mathrm{~nm}$. It follows a thick shell of about $500 \mathrm{~nm}$ with round pores of size in the range of $10 \mathrm{~nm}$ (P2 region), and another $100 \mathrm{~nm}$ thick shell with pores of typical size of $1 \mathrm{~nm}$ (P3 region). Finally, as mentioned above, the outermost layer (NPS) is non-porous, and its thickness is about $100 \mathrm{~nm}$. 
In spite of the internal porous structure, the FESEM images of the outside aspect (Fig. 3 (b), (c)) of the NPS show it is highly spherical and with a smooth surface. Therefore, although modes, like $b_{13,5}$, are killed by the porous structure, other type of resonances, which are more localized near the NPS and at the microsphere's surface, like those with high number and low order mode, could in principle resonate. Our scattering setup has not been able to detect such high $Q$ modes, but they could be detected by more sensitive approaches. Moreover, they might be the origin of the rich absorption spectra observed by measuring the electronic response in crystallized and doped silicon microspheres [14]. This would prove, at the same time, the high sensitivity of this technique, consisting of measuring absolute absorption (photocurrent) rather than scattering at a defined angle.

The origin of such a porous structure constitutes currently an object of research. Nevertheless, here we hypothesize that it may be originated from the high content of hydrogen in the as-synthesized amorphous silicon microspheres, which comes from the precursor gas, $\mathrm{Si}_{2} \mathrm{H}_{6}$. If the crystallization process is relatively fast, the outermost shell of an amorphous particle crystallizes before the hydrogen from inside of the particle can be degassed. As a result, the remaining hydrogen may evolve into bubbles during the atomic rearrangement. We believe that the size of the pores is related to the amount of trapped hydrogen and to the particle formation process, where micrometer size particles are formed by coalescence of smaller ones [34], in a similar manner to what happens with colloidal synthesis of $\mathrm{SiO}_{2}$ by the Stöber method in liquid suspensions [35].
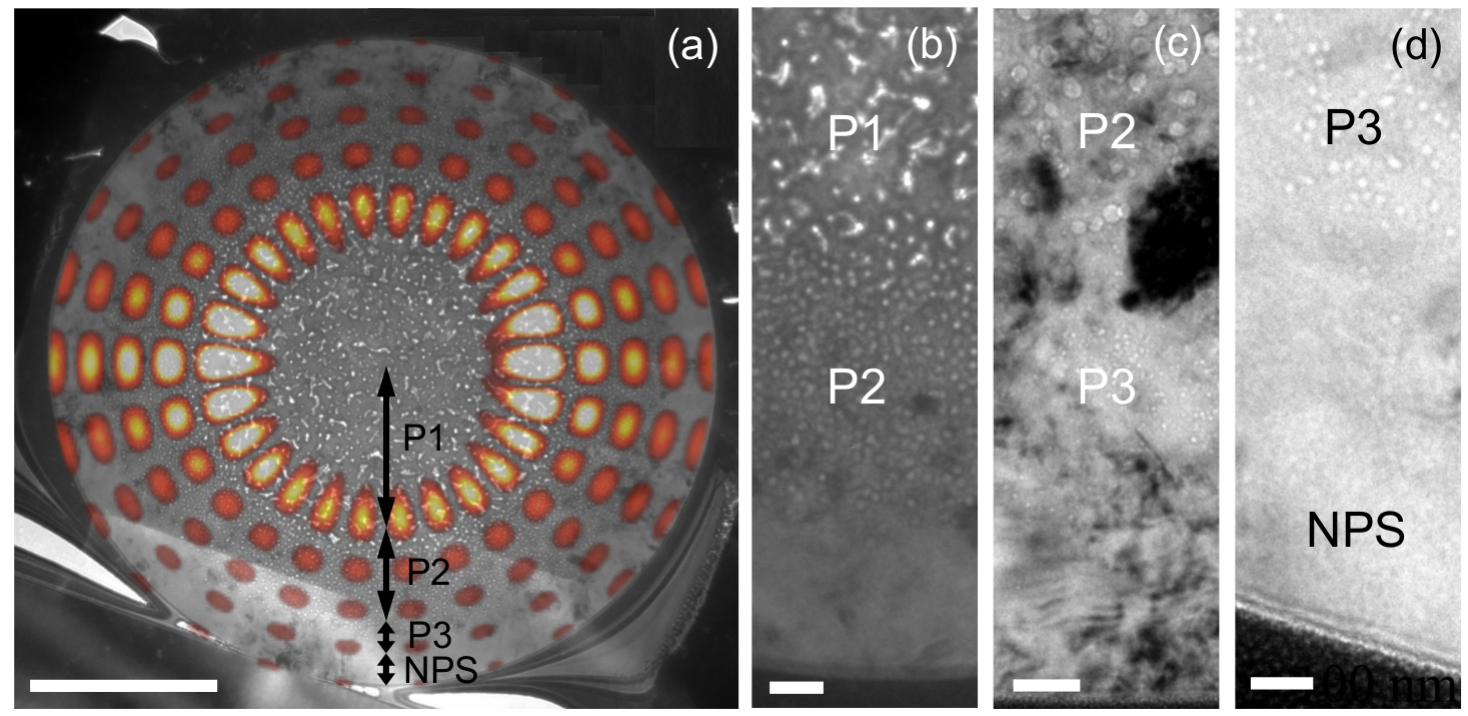

FIG. 8. (Color online) (a) HRTEM image of the internal surface of a fast crystallized silicon microsphere, superimposed by the electric field intensity distribution of mode $b_{13,5}$. The positions of the different porous regions: P1, P2 and P3, as well as the non-porous shell (NPS) are indicated. (b) Zoomed area of (a) for better visualizing the transition between P1 and P2. (c) and (d) Higher magnification images illustrating the transition between P2-P3 and P3NPS respectively. The scale bars correspond to $1 \mu \mathrm{m}$ (a), $100 \mathrm{~nm}$ (b), $50 \mathrm{~nm}$ (c) and $20 \mathrm{~nm}(\mathrm{~d})$. 

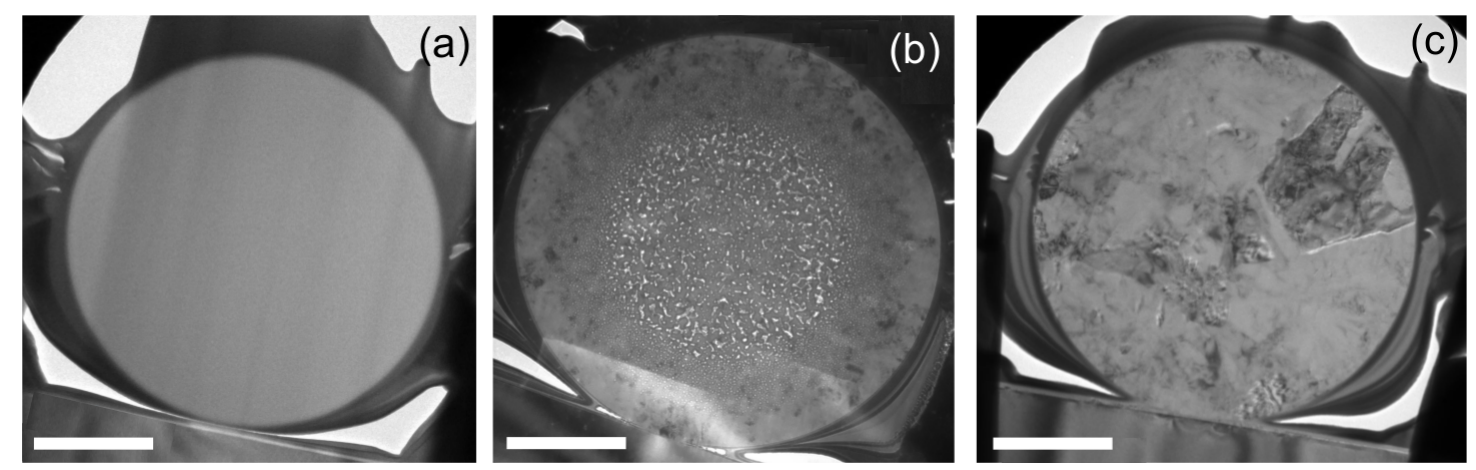

FIG. 9. HRTEM images of the inside surface of amorphous (a), and polycrystalline silicon microspheres obtained by the fast (b) and the slow (c) crystallization regimes. The scale bars correspond to $1 \mu \mathrm{m}$.

In contrast to the observed porous structure in fast crystallized silicon microspheres, the inside surface of an amorphous silicon microsphere is very smooth and free of pores and defects (see Fig 9 (a)). Therefore, it constitutes, in principle, a much more suited platform for observing high $Q$ resonant phenomena. Figure 9 (b) shows again the inside structure of the fast crystallized microsphere for comparison. On the other hand, the slow crystallization process preserves the pronounced resonances in the scattering spectra much better than the fast crystallization regime (see Fig. 4 (a) and (b)). The observation of the inside structure of slowly crystallized microspheres revealed that this fact is related to the suppression of pores (see Fig. 9 (c)). Indeed, the slow heating process should favour an easier release of hydrogen from the inner part of the particles, thus allowing a better reorganization of the silicon atoms in the crystal lattice. However, other defects like those related to the finite size of the crystal domains, which can be guessed by the uniform greyed areas, are still present.

The scattering spectra of slowly crystallized microspheres could be fitted fairly well to simulations given by Mie theory. In this case, at difference to the amorphous case, only the sphere diameter was considered as a fitting parameter because the refractive index values of crystalline silicon do not depend on the fabrication method. Fig. 10 is an example of such a fit for sphere \#6 of Table I. We used, for the real part of the refractive index, values compiled by Palik [31] in the wavelength range from $500 \mathrm{~nm}$ to $1200 \mathrm{~nm}$, and values compiled by Li [36] form $1200 \mathrm{~nm}$ to $1600 \mathrm{~nm}$. Regarding the imaginary part, we used the values reported by $\mathrm{R}$. Braunstien et al. [37] for intrinsic silicon for wavelengths shorter than $1150 \mathrm{~nm}$, assuming a value of zero for longer wavelengths. This makes, logically, that many high- $Q$ resonances appear in the simulated curve at the longest wavelength region, where no absorption is considered, but that can not be detected experimentally because of the same setup limitations explained above for the case of amorphous microspheres. Still, such high- $Q$ resonances might be there and would be influenced by the structural defects as well and, therefore, should be further investigated with the appropriate techniques. In any case, the assumption of standard refractive index values led to fits of sphere diameters with very small errors, of the order of several nanometers (fourth column in Table I), which correspond to remarkable relative errors of about $0.1 \%$. These errors are 
originated from the small deviations in the position between the simulated and the experimental peaks that occur at a given diameter value. They were calculated as the difference between the maximum and the minimum diameter that is required for any simulated resonance of the spectrum to match its corresponding experimental peak. This contrasts the much bigger errors, obtained from electron microscopy images (third column in Table I), and it is an order of magnitude smaller than the usual size dispersion of the particles used for size calibration purposes. Therefore, crystallized silicon microspheres, together with the reported optical scattering characterization method plus the fitting process to Mie theory, could find application as accurate size calibration standards.

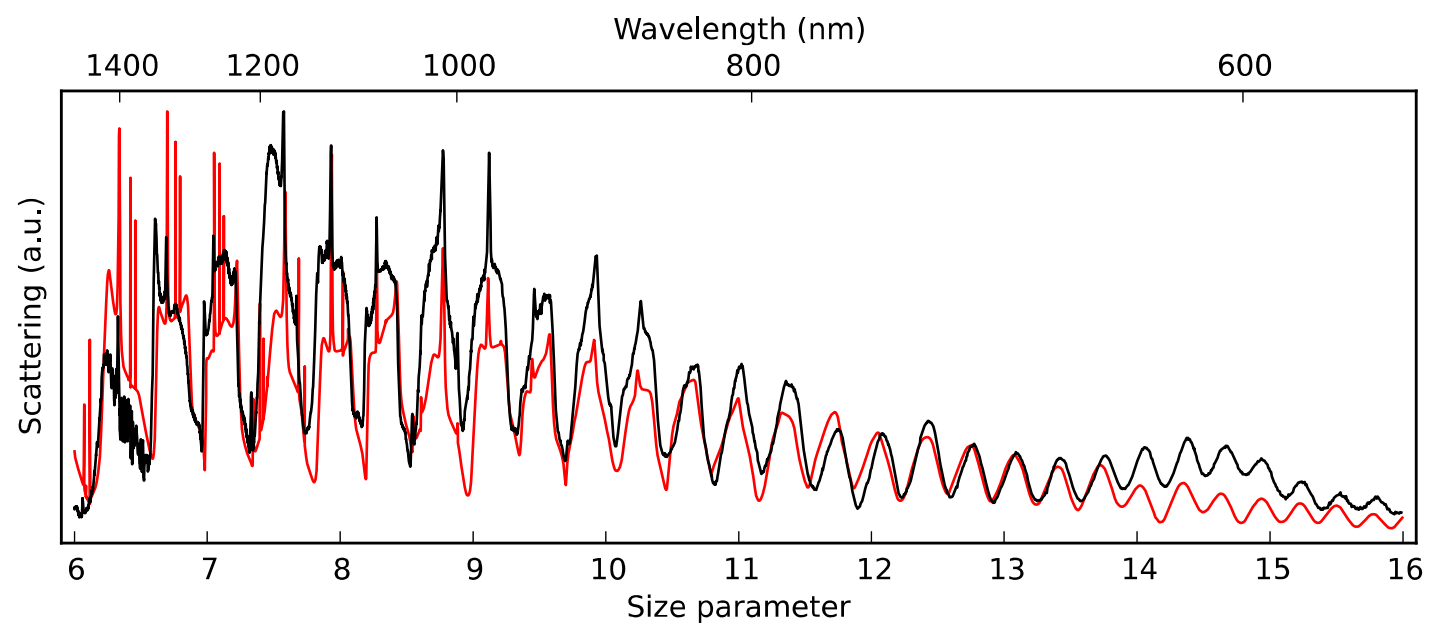

FIG. 10. (Color online) Experimental (black curve) and simulated ((red) grey curve) spectra for microsphere 6 of Table I in the crystalline state. The spectra are plotted against the wavelength of light, $\lambda$ (top axis) and the size parameter (bottom axis), defined as $\pi * d / \lambda$, where $d$ is the sphere diameter.

\section{CONCLUSIONS}

Silicon microspheres behave as optical microcavities in their amorphous as well as in their poly-crystalline phase because of their good spherical condition, surface smoothness, and the high refractive index of the material. Optical scattering at $90^{\circ}$ has been proven to be a very convenient technique to measure and analyse their resonances. Two regimes of crystallization were studied, a fast one and a slow one, and no conclusive external changes, regarding sphericity and surface smoothness, were observed between the amorphous and the crystalline particles. However, we found substantial differences in their optical scattering spectra, which were attributed to changes in their internal structure. Fast crystallized microspheres have an internal radially distributed porous structure, with pores size in the range from 100 to $1 \mathrm{~nm}$, surrounded by a non-porous shell. This makes fade out those high- $Q$ resonances whose electromagnetic field is well distributed along the porous regions. On the other hand slowly crystallized microspheres did not show any pores and could better sustain pronounced modes. In both cases, they have defects related to their poly-crystalline nature that can diminish the 
quality of the resonances. The scattering spectra of amorphous and crystallized microspheres could be fitted fairly well to simulated spectra obtained by applying Mie theory, and remarkable errors of about $0.1 \%$, below the error obtained through FESEM imaging, were obtained for the fitted diameters in the last case.

\section{ACKNOWLEDGEMENTS}

This work was supported by projects ENE2013-49984-EXP (Spanish Ministry of Economy and Competitiveness, MINECO), MAT2012-35040, and PROMETEOII/2014/026. The authors greatly acknowledge the Electron Microscopy Service of the UPV for their valuable help in the structural characterization of the microspheres.

1 F. Meseguer, R. Fenollosa, I. Rodríguez, E. Xifré-Pérez, F. Ramiro-Manzano, M. Garín, and M. Tymczenko, J. Appl. Phys. 109, 102424 (2011).

2 A. García-Etxarri, R. Gómez-Medina, L.S. Froufe-Pérez, C. López, L. Chantada, F. Scheffold, J. Aizpurua, M. Nieto-Vesperinas and J. J. Sáenz, Opt. Exp. 19, 4815 (2011).

3 A. B. Evlyukhin, S. M. Novikov, Urs. Zywietz, R. L. Eriksen, C. Reinhardt, S. I. Bozhevolnyi, and B. N. Chichkov, Nanoletters 12, 3749 (2012).

4 A. E. Krasnok, A. E. Miroshnichenko, P. A. Belov, and Y. S. Kivshar, Opt. Exp. 20, 20599 (2012).

5 M. I. Tribelsky, J.-M. Geffrin, A. Litman, C. Eyraud, and F. Moreno, Sci. Rep. 5, 12288 (2015).

6 J. M. Jasinski and S. M. Gates, Acc. Chem. Res. 24, 9 (1991).

7 F. García-Santamaría, M. Ibisate, I. Rodríguez, F. Meseguer, and C. López, Adv. Mater. 15, 788 (2003).

8 Handbook of Semiconductor Silicon Technology (Ed. W.C. O'Mara, R.B. Herring, L.P. Hunt, Noyes Pub. 1990).

9 R. Fenollosa, F. Meseguer, and M. Tymczenko, Adv. Mater. 20, 95 (2008).

10 E. Xifré-Pérez, J. D. Domenech, R. Fenollosa, P. Muñoz, J. Capmany, and F. Meseguer, Opt. Exp. 19, 3185 (2011).

11 R. Paniagua-Domínguez, F. López-Tejeira, R. Marqués, and J. A. Sánchez-Gil, New J. Phys. 13, 123017 (2011).

12 A. I. Kuznetsov, A. E. Miroshnichenko, Y. H. Fu, J. Zhang, and B. Luk'yanchuk, Sci. Rep. 2, 492 (2012).

13 L. Shi, J. T. Harris, R. Fenollosa, I. Rodriguez, X. Lu, B. A. Korgel, F. Meseguer, Nat. Comm. 4, 1904 (2013).

14 M. Garín, R. Fenollosa, R. Alcubilla, L. Shi, L.F. Marsal, and F. Meseguer, Nat. Comm. 5, 3440 (2014).

15 S.-C. Hung, S.-C. Shiu, C.-H. Chao, and C.-F. Lin, J. Vac. Sci. Technol. B, 27, 1156 (2009).

16 J. T. Harris, J. L. Hueso, and B.K. Korgel, Chem. Mater. 22, 6378 (2010). 
17 X. Li, A. Pyatenko, Y. Shimizu, H. Wang, K. Koga, and N. Koshizaki, Langmuir 27, 5076 (2011).

18 U. Zywietz, A. E. Evlyukhin, C. Reinhardt, and B. N. Chichkov, Nat. Comm. 5, 3402 (2014).

19 E. Xifré-Pére, R. Fenollosa, and F. Meseguer, Opt. Exp. 19, 3455 (2011).

20 Q. Shabir, A. Pokale, A. Loni, D. R. Johnson, L. T. Canham, R. Fenollosa, M. Tymczenko, I. Rodriguez, F. Meseguer, A. Cros, and A. Cantarero, Silicon, 3, 173 (2011).

21 R. Fenollosa, E. Garcia-Rico, S. Alvarez, R. Alvarez, X. Yu, I. Rodriguez, S. CarregalRomero, C. Villanueva, M. Garcia-Algar, P. Rivera-Gil, A. R. de Lera, W. J. Parak, F. Meseguer, and R. A. Alvarez-Puebla, J. Nanobiotech. 12, 35 (2014).

22 C. Spinella and S. Lombardo, J. Appl. Phys. 84, 5383 (1998).

23 J. Farjas, C. Rath, P. Roura, and P. Roca i Cabarrocas, Appl. Surf. Sci. 238, 165 (2004).

24 L. Shi, R. Fenollosa, T. Umut Tuzer, and F. Meseguer, ACS Photonics, 1, 408 (2014).

25 A. Ashkin and J.M. Dziedzic, Appl. Opt. 20, 1803 (1981).

26 J. M. Ramírez, D. Navarro-Urrios, N.E. Capuj, Y. Berencén, A. Pitanti, B. Garrido, and A. Tredicucci, Sci. Rep. 5, 14452 (2015).

27 C. F. Bohren and D. R. Huffman, Absorption and Scattering of Light by Small Particles ( John Wiley \& Sons, New York 1998).

28 P. W. Barber and S. C. Hill, Light Scattering by Particles: Computational Methods, (World Scientific, Singapore, 1990).

29 P.R. Conwell, P.W. Barber, and C.K. Rushforth, J. Opt. Soc. Am. A, 1, 62 (1984).

30 R. I. Badran, F.S. Al-Hazmi, S. Al-Heniti, A.A. Al-Ghamdi, and J. Li, S. Xiong, Vacuum 83, 1023 (2009).

31 E. Palik, Handbook of Optical Constants of Solids, Vol. 1 (Academic Press, New York, 1985).

32 D. T. Pierce and W. E. Spicer, Phys. Rev. B, 5, 3017 (1972).

33 M. Garín, R. Fenollosa, P. Ortega, and F. Meseguer, J. Appl. Phys. 119, 033101 (2016).

34 R. Kömer, H. -J. Schmid, and W. Peukert, J. Aeros. Sci. 41, 1008 (2010).

35 W. Stöber, A. Fink, and E. Bohn, J. Colloid Interface Sci. 26, 62 (1968).

36 H. H. Li, J. Phys. Chem. Ref. Data, 9, 561 (1980).

37 R. Braunstein, A. R. Moore, and F. Herman, Phys. Rev. 109, 695 (1958). 